SLAC-PUB-10524

June 2004

\title{
The Sub-parsec, Geometrically Thick, Self-Gravitating Accretion Disk in the Nucleus of NGC 3079
}

\author{
Paul T. Kondratko, L. J. Greenhill, and J. M. Moran \\ Harvard-Smithsonian Center for Astrophysics, \\ Cambridge, MA 02138, USA, \\ email: pkondrat@cfa.harvard.edu
}

\footnotetext{
Contributed to the International Astronomical Union Symposium 222, The Interplay Among Black Holes, Stars, and ISM in Galactic Nuclei,

3/1/2004-3/5/2004, Gramado/Rio Grande do Sul/Brazil
} 


\title{
The Sub-parsec, Geometrically Thick, Self-Gravitating Accretion Disk in the Nucleus of NGC 3079
}

\author{
Paul T. Kondratko, L. J. Greenhill, and J. M. Moran \\ Harvard-Smithsonian Center for Astrophysics, Cambridge, MA 02138, USA, email: \\ pkondrat@cfa.harvard.edu
}

\begin{abstract}
We have mapped, for the first time, the full velocity extent of the water maser emission in NGC 3079. Based on the analysis of the spectral-line maps, we propose a nearly edge-on, massive, thick, and flared disk for the geometric model of the inner parsec. The disk orbits a mass of $\sim 2 \times 10^{6} M_{\odot}$ enclosed within $0.4 \mathrm{pc}$, most likely a supermassive black hole. The disk is most likely self-gravitating, clumpy, and supportive of star formation. The presence in our VLBI continuum images of an aging synchrotron component, which is not collinear with the previously imaged jet, might be suggestive of changes in jet orientation. The jet may coexist on pc-scales with a wide-angle outflow, that is related to the kpc-scale superbubble and that is inferred from the observation of dense molecular material at high latitudes above the disk.
\end{abstract}

NGC 3079 is a nearby $\left(v_{\text {sys }}=1125 \mathrm{~km} \mathrm{~s}^{-1}\right)$, nearly edge-on galaxy that harbors an AGN, an alleged nuclear starburst, and one of the most luminous water masers $(\lambda=$ $1.3 \mathrm{~cm}$ ) known. Along the minor axis of the galaxy, a bipolar super-wind inflates a kpcscale superbubble observed in many wavebands (Fig. 1).

In a recent VLBI experiment, we mapped, for the first time, the full velocity extent of the water maser emission in NGC 3079. The largely north-south distribution of emission, aligned with a known kpc-diameter molecular disk, and the segregation of blue and redshifted emission on the sky are suggestive of a nearly edge-on molecular disk on pc-scales (Fig. 1). Positions and line-of-sight velocities of blue and red-shifted maser emission are consistent with a central mass of $\sim 2 \times 10^{6} M_{\odot}$ enclosed within a radius of $\sim 0.4 \mathrm{pc}$. The corresponding mean mass density of $10^{6.8} M_{\odot} \mathrm{pc}^{-3}$ is suggestive of a central black hole, which is consistent with the detection of hard X-ray excess $(20-100 \mathrm{keV})$ and an Fe K $\alpha$ line in the direction of the nucleus (Iyomoto et al. 2001; Cecil et al. 2002). For a 40 to $160 \mathrm{~km} \mathrm{~s}^{-1}$ stellar bulge velocity dispersion (Shaw et al. 1993), the enclosed mass is consistent with correlations between central mass and dispersion reported for broad samples of galaxies (e.g., Gebhardt et al. 2000). The ratio of X-ray (Iyomoto et al. 2001) to Eddington luminosity is $0.004<\epsilon<0.04$, consistent with accretion efficiencies of $0.01 \leqslant \epsilon \leqslant 1$ obtained for Seyfert 1 systems (Padovani 1989). Because the rotation curve traced by the maser emission is flat, the mass of the circumnuclear disk is significant with respect to the central mass. Since the velocity dispersion of the maser features does not decrease with radius and the velocity differences between neighboring maser features (tens of $\mathrm{km} \mathrm{s}^{-1}$ ) are a significant fraction of the $\sim 150 \mathrm{~km} \mathrm{~s}^{-1}$ orbital velocity, the disk is probably thick and flared. The physical conditions necessary to support maser emission imply a Toomre $Q$-parameter that is $<1$. Thus, the disk is probably clumpy and it is these discrete clumps of matter heated by X-ray emission from the central engine that probably give rise to the maser emission. The Jean's mass, the Roche limit, the cooling timescale, and the clump collision frequency are all suggestive of star formation in the disk. Overall, the accretion disk in NGC 3079, a model of which is shown in Fig.1, stands 


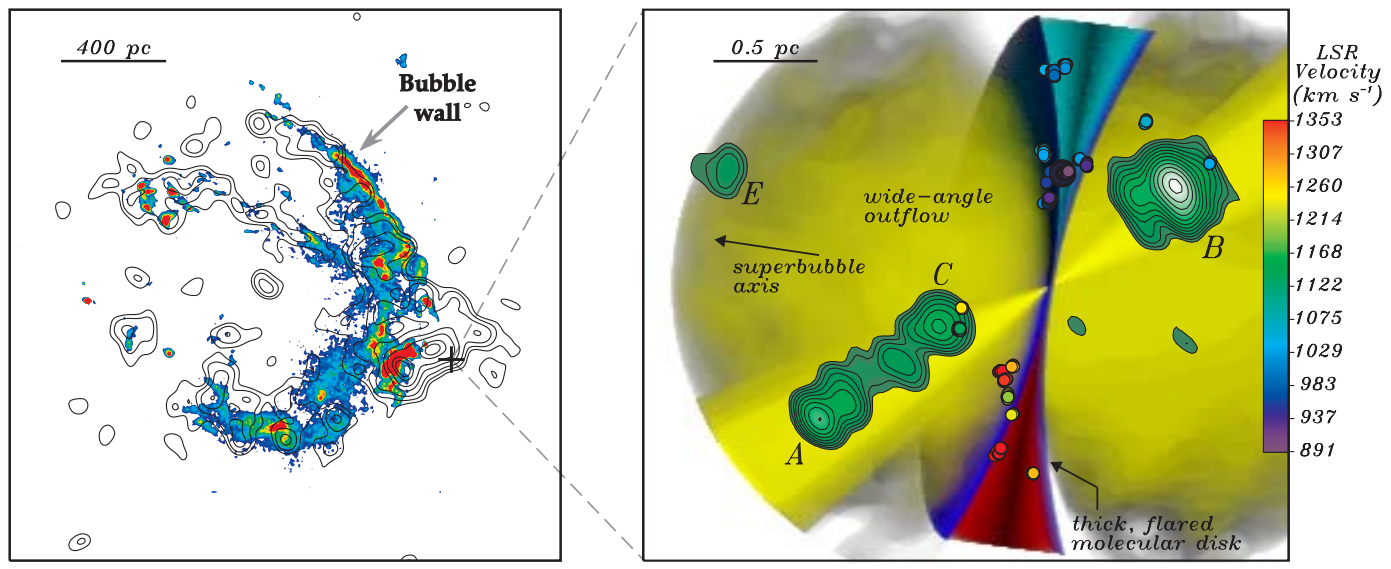

Figure 1. (left) $[\mathrm{NII}]+\mathrm{H} \alpha$ image of the superbubble (filled color contours) obtained with the HST (Cecil et al. 2001) and the soft X-ray image of the same (unfilled black contours) obtained with the Chandra X-ray Observatory (CXO). The cross indicates both the VLBI-derived position of the maser source with respect to the X-ray emission as well as the $0.6^{\prime \prime}$ uncertainty in the CXO absolute astrometry. (right) Proposed model for the nuclear region of NGC 3079, a VLBI image of $8 \mathrm{GHz}$ continuum (green contours), and a map of $22 \mathrm{GHz}$ maser emission (shown as spots color-coded by Doppler velocity). We propose a thick and flared disk (shown in a cross-section) that is slightly tilted with the west side being closer and that is aligned with both the kpc-scale molecular disk and the axis of the kpc-scale superbubble. The color coding indicates the Doppler shift of the disk material. The water maser emission traces the disk and may arise from X-ray irradiated clumps within the disk, which is unstable to fragmentation. An off-axis jet, traced by the continuum components $\mathrm{A}, \mathrm{B}$, and $\mathrm{C}$, probably coexists with a wide-angle outflow (yellow), most likely an inward extension of the kpc-scale superbubble. The outflow may result in an uplift of clumps from the disk surface, which would explain the high latitude maser features and their association in velocity with the most proximate side of the disk.

in contrast to the compact, thin, warped, differentially rotating disk in the archetypal maser galaxy NGC 4258 .

We have also mapped with VLBI radio continuum emission in the vicinity of the disk and identify a new, time-variable, non-thermal component (E) that is not collinear with the previously imaged putative jet, traced by components A, B, and C (Fig.1). Based on the large luminosity and the unusually steep spectrum $(\alpha<-2.1)$, we exclude a radio supernova as the progenitor of E. However, because its spectrum is consistent with an aging electron energy distribution, E might be a rapidly cooling remnant, indicating that the jet axis wobbles. Alternatively, considering its location within the opening angle of the kpc-scale superbubble, the component might mark a shock in a wide-angle outflow that is interacting with a dense ambient medium. In this context, molecular gas at high latitudes above the disk, mapped in this and previous studies (i.e., the two eastermost and two westernmost maser features in Fig. 1), may be a part of a pc-scale wide-angle outflow, likely an inward extension of the kpc-scale superbubble.

\section{References}

Cecil, G., Bland-Hawthorn, J., \& Veilleux, S. 2002, ApJ, 576, 745

Cecil, G., Bland-Hawthorn, J., Veilleux, S., \& Filippenko, A. V. 2001, ApJ, 555, 338

Gebhardt, K., et al. 2000, ApJ, 539, L13

Iyomoto, N., Fukazawa, Y., Nakai, N., \& Ishihara, Y. 2001, ApJ, 561, L69

Padovani, P. 1989, A\&A, 209, 27

Shaw, M., Wilkinson, A., \& Carter, D. 1993, A\&A, 268, 511 